\title{
Inclusión de grasa en dieta de cerdas primíparas: efecto sobre la reproducción y performance de la camada
}

\author{
Rivas, F.R. ${ }^{1,2}$; Gonzalez, M.A. ${ }^{2}$; Matiller, V.; Williams, S.I. ${ }^{4}$ \\ ${ }^{1}$ Consejo Nac. Investig. Científ. \& Técn. (CONICET). ${ }^{2}$ INTA Las Breñas, Chaco, Argentina. \\ ${ }^{3}$ Lab Biol. Cel.y Molec. (Univ. Nac. Litoral, UNL), Esperanza, Santa Fe, Argentina. \\ ${ }^{4}$ Fac.Cs.Vet. UNLP, La Plata, Argentina. E-mail: rivas.francisco@inta.gob.ar
}

\begin{abstract}
Resumen
Rivas, F.R.; Gonzalez, M.A.; Matiller, V.; Williams, S.I.: Inclusión de grasa en dieta de cerdas primíparas: efecto sobre la reproducción y performance de la camada. Rev. Vet. 31: 1, 61-65, 2020. La incorporación de grasa en la dieta podría mejorar la performance reproductiva de cerdos en producción, principalmente durante las épocas más calurosas. Para comprobar esta hipótesis se evaluó el efecto de la inclusión de grasa a diferentes niveles sobre variables reproductivas de cerdas primíparas en condiciones de subtrópico. Se utilizaron 3 grupos de 6 animales $(n=18)$ a los cuales se les suministraron 3 dietas con diferentes niveles de inclusión de grasa $(0 \%, 3,5 \%$ y $7 \%)$ desde el día 90 de gestación y durante la lactación (21 días). Las variables medidas fueron el número de lechones nacidos totales, nacidos vivos, muertos y peso de la camada al nacimiento y al destete, mientras que sobre las hembras se registró el intervalo de retorno al celo. Se empleó un diseño completamente aleatorizado y los datos fueron analizados mediante ANOVA con el software estadístico Infostat ${ }^{\circledR}$. No se encontraron diferencias al incorporar grasa, en relación al número de lechones nacidos vivos, muertos, totales, peso al nacimiento e intervalo-destete celo $(\mathrm{p}>0,05)$ mientras que el número y peso de lechones al destete se vio influenciado de manera significativa $(\mathrm{p}<0,05)$. En conclusión, la adición de grasa en dietas de cerdas primíparas no afecta variables al nacimiento ni el intervalo detete-celo, pero mejora el número y peso de los lechones al destete.
\end{abstract}

Palabras clave: cerdas, lípidos, nutrición, peso vivo, reproducción, estrés calórico.

\begin{abstract}
Rivas, F.R.; Gonzalez, M.A.; Matiller, V.; Williams, S.I.: Inclusion of fat in the diet of primiparous sows: effect on the reproduction and litter performance. Rev. Vet. 31: 1, 61-65, 2020. Adding fat to sow diets may improve reproductive performance during the warm season. Therefore, the objective of this study was to evaluate the effect of fat addition at different levels on reproductive variables of primiparous sows in subtropical conditions. Three groups of six animals $(\mathrm{n}=18)$ were fed with three diets with different fat levels $(0 \%, 3.5 \%$ and $7 \%)$ from day 90 of gestation until the end of lactation (21 days). Litter size, weight and number of piglets born alive and stillborn were recorded, as well as weight and number of pigs at weaning, along with weaning-to-estrus interval. Completely random design was used, and collected data were analyzed using Infostat ${ }^{\circledR}$ statistical software. ANOVA was used for means comparison. No differences between treatments were observed among number and weight of pig born alive, stillborn, litter size and weaning-to-estrus interval ( $p>0.05)$, whereas number and weight of pigs at weaning were increased by the addition of fat $(\mathrm{p}<0.05)$. In conclusion, fat inclusion resulted in an improved number and weight of piglets at weaning; however, weaning-to-estrus and variables at birth, were not affected.
\end{abstract}

Key words: sows, lipids, nutrition, liveweight, reproduction, heat stress,

\section{INTRODUCCIÓN}

El estrés calórico es uno de los principales problemas que afectan la eficiencia de la producción porcina no solo en países subtropicales sino también en aquéllos de clima templado ${ }^{18}$. En Estados Unidos las pér-

Recibido: diciembre 2019 / Aceptado: abril 2020 didas económicas por estrés calórico ascienden a 330450 millones de dólares anuales ${ }^{13,14}$.

El cerdo, al igual que otros mamíferos homeotermos, debe regular su temperatura corporal para mantener un balance entre la generación y la pérdida de calor. Debido a su baja capacidad para disipar el calor por medios físicos, los cerdos sometidos a altas temperaturas $\left(>25^{\circ} \mathrm{C}\right)$ reducen su consumo voluntario de 
alimento como principal mecanismo para disminuir la producción de calor metabólico ${ }^{17,23}$.

Por su elevado metabolismo durante la lactación y etapas tardías de gestación, las hembras porcinas (principalmente las primíparas) son las más susceptibles a los efectos negativos de las altas temperaturas ${ }^{25}$. A ello hay que sumarle que las cerdas reproductoras modernas están seleccionadas por su alta prolificidad y producción de leche asociadas a un intenso metabolismo y bajo consumo de alimento, lo cual las torna más sensibles al calor ${ }^{6}$.

La reducción del consumo conduce al incremento de la movilización de reservas corporales ocasionando problemas reproductivos tanto en la hembra gestante (alta mortalidad embrionaria y aumento del número de lechones nacidos muertos, entre otros) como en la hembra en lactancia (baja producción de leche, disminución de la performance de la camada y la subsecuente performance reproductiva de la hembra) ${ }^{2,3,8,15}$.

La inclusión de grasa en dietas de cerdas puede reducir los efectos negativos del estrés calórico debido a su alta densidad energética y bajo incremento calórico, asociados a su adecuada digestión y metabolismo ${ }^{19,20}$.

La evidencia sugiere que la adición de grasa durante la gestación tardía ( $>90$ días) y la lactación, aumentaría la producción de leche, la ganancia de peso de los lechones y su supervivencia hasta el destete ${ }^{1,16}$, a la vez de reducir el intervalo destete-celo ${ }^{12}$. Sin embargo, los trabajos que estudian el efecto de la suplementación con grasa a diferentes niveles bajo altas temperaturas, son escasos ${ }^{22}$.

En relación a lo expuesto anteriormente, el objetivo de este trabajo fue evaluar el efecto de la inclusión de grasa en diferentes niveles sobre la performance reproductiva de cerdas primíparas y sus camadas, en un sistema de producción subtropical.

\section{MATERIAL Y MÉTODOS}

Los procedimientos aquí empleados fueron aprobados por el Comité Asesor de Ética y Seguridad de la Facultad de Veterinaria de la Universidad Nacional del Litoral (Expte. 0898035-17).

Respecto a las instalaciones y manejo de animales, un total de 18 hembras primíparas (Landrace $\mathrm{x}$ Yorkshire) fueron alojadas en una granja porcina semiintensiva en la localidad de Concepción del Bermejo, Chaco, Argentina. Las hembras estaban reunidas en grupos de 6 animales cada uno, según la cuota semanal de servicio del establecimiento. El peso promedio
Tabla 2. Composición y valores energéticos de la grasa empleada* para las dietas experimentales.

\begin{tabular}{lc}
\hline composición & \\
\hline humedad máxima (\%) & 3,5 \\
grasa bruta (\%) & 84,0 \\
cenizas (\%) & 12,6 \\
calcio (\%) & 9,0 \\
\hline ácidos grasos & 0,2 \\
\hline ac. mirístico (C14:0) (\%) & 11,4 \\
ac. palmítico (C16:0) (\%) & 4,6 \\
ac. esteárico (C18:0) (\%) & 23,5 \\
ac. oleico (C18:1) (\%) & 52,0 \\
ac. linoleico (C18:2) (\%) & 7,7 \\
\hline valores energéticos & 6,1 \\
\hline energía bruta (Mcal/kg MS)
\end{tabular}

* grasa protegida proveniente de aceites vegetales e hidróxido de calcio.

al momento del servicio fue de $160 \pm 3 \mathrm{~kg}$ y todas las cerdas presentaron el mismo estado sanitario.

Para garantizar la manifestación de celo de las cerdas de cada grupo, se utilizó un protocolo de sincronización de celo mediante Altrenogest $0.4 \%$ durante 18 días, esperando síntomas de celo aproximadamente el día 5 post-retiro del tratamiento.

Las hembras fueron servidas en sus respectivas jaulas de gestación $(2,4 \times 0,6 \mathrm{~m})$ mediante inseminación artificial con semen proveniente de un macho dador del propio establecimiento. El día 110 de gestación las 
Tabla 3. Efecto de la inclusión de grasa sobre variables al nacimiento y destete.

\begin{tabular}{lccccc}
\hline variable & $\operatorname{Tr} 0 \%$ & $\operatorname{Tr} 3,5 \%$ & $\operatorname{Tr} 7 \%$ & $\mathrm{EE}$ & P-valor \\
\hline número de cerdas & 6 & 6 & 6 & - & - \\
lechones nacidos vivos & $10,7^{\mathrm{a}}$ & $11,3^{\mathrm{a}}$ & $12,7^{\mathrm{a}}$ & 1,09 & 0,4349 \\
lechones nacidos muertos & $2,20^{\mathrm{a}}$ & $1,20^{\mathrm{a}}$ & $0,80^{\mathrm{a}}$ & 0,65 & 0,3504 \\
lechones nacidos totales & $12,8^{\mathrm{a}}$ & $12,5^{\mathrm{a}}$ & $14,0^{\mathrm{a}}$ & 1,16 & 0,6375 \\
peso promedio nacimiento $(\mathrm{kg})$ & $1,34^{\mathrm{a}}$ & $1,43^{\mathrm{a}}$ & $1,37^{\mathrm{a}}$ & 0,09 & 0,7322 \\
lechones destetados & $10,0^{\mathrm{a}}$ & $12,3^{\mathrm{b}}$ & $12,3^{\mathrm{b}}$ & 0,70 & 0,0509 \\
peso promedio al destete (kg.) & $4,95^{\mathrm{a}}$ & $5,72^{\mathrm{b}}$ & $5,81^{\mathrm{b}}$ & 0,24 & 0,0450 \\
intervalo destete cerdo (días) & $5,50^{\mathrm{a}}$ & $5,17^{\mathrm{a}}$ & $5,00^{\mathrm{a}}$ & 0,16 & 0,1100 \\
\hline
\end{tabular}

Tr: tratamiento. EE: error estándar. En la misma hilera, medias con diferente superíndice difieren significativamente $(\mathrm{p}<0,05)$.

hembras fueron trasladadas a las jaulas de maternidad ( $2,4 \times 0,9 \mathrm{~m})$, donde permanecieron hasta el momento del destete (21 días). La temperatura en el interior de las instalaciones fue regulada mediante cortinas y sistema de goteo.

Para la determinación del intervalo destete-celo (IDC), las hembras fueron trasladadas a las jaulas de gestación el día posterior al destete. La presentación de celo fue monitoreada dos veces al día (mañana y tarde) mediante el estímulo de un macho detector. Se consideró como celo verdadero el reflejo de inmovilización de la hembra (presión sobre espalda). Todos los animales tenían libre acceso al agua por medio de bebederos tipo chupetes.

En cuanto a la alimentación, los tratamientos estuvieron conformados por una dieta de gestación y de lactación sin agregado de grasa ( $\mathrm{G} 0$ y L0), una dieta de gestación y de lactación con inclusión de 3,5\% de grasa (G1 y L1) y una dieta de gestación y de lactación con $7 \%$ de grasa (G2 y L2).

Desde el primer día de gestación y hasta el día 90 todas las hembras consumieron la dieta $\mathrm{G} 0$ a razón de $2,5 \mathrm{~kg} / \mathrm{animal} / \mathrm{día}$. Desde el día 91 y hasta el parto se asignaron al azar las dietas G1 y G2 a grupos de igual número de individuos $(\mathrm{n}=12)$ mientras que el grupo (control) continuó con la dieta G0 $(\mathrm{n}=6)$. Durante este periodo el alimento fue suministrado a razón de $3 \mathrm{~kg} /$ animal/día dividido en dos tomas diarias (8 y $16 \mathrm{~h}$ ).

Luego del parto y hasta el nuevo servicio las hembras consumieron las dietas L0, L1 y L2 coincidentes con la dieta asignada en gestación. Durante este periodo las dietas fueron suministradas a discreción.

Todas las dietas empleadas fueron formuladas para que cumplan o superen las recomendaciones nutricionales ${ }^{9}$. Aquellas dietas con agregado de grasa se mantuvieron isocalóricas. La forma de presentación del alimento para ambas etapas (gestación y lactación) fue molido fino. La composición de las dietas experimentales, así como de la grasa empleada se detallan en las Tablas 1 y 2 respectivamente.

En las salas de gestación y maternidad, la temperatura ambiental $\left({ }^{\circ} \mathrm{C}\right)$ y la humedad relativa (\%) se controlaron mediante el uso de termo-higrómetros di- gitales (Datalogger DTL2) de lectura y registro automático (a intervalos de $1 \mathrm{~h}$ ).

En cuanto al diseño experimental y análisis estadístico, se registró el tamaño total de la camada (LNT), número y peso de lechones nacidos vivos (LNV) y lechones muertos (LNM). Cuarenta y ocho horas luego del parto se procedió a la homogeneización de las camadas (crossfostering) dentro de cada tratamiento, para garantizar la mínima diferencia en el tamaño de las camadas ( \pm 1 lechón). El número y peso de lechones destetados, así como el intervalo destete-celo, también fueron registrados.

Se empleó un diseño completamente aleatorizado y los datos fueron analizados empleando el método de modelos lineales y mixtos de Infostat v. 2018. Se testearon los supuestos de normalidad y homocedasticidad mediante $Q-Q$ Plot y gráfico de residuos vs predichos respectivamente. El modelo general empleado fue el siguiente: $\mathrm{y}_{\mathrm{ij}}=\mu+\alpha_{\mathrm{i}}+\varepsilon_{\mathrm{ij}}(\mathrm{y}=$ variable respuesta, $\mu=$ media general, $\alpha_{i}=$ efecto del tratamiento, $\varepsilon_{i j}=$ error aleatorio). Se empleó un ANOVA para detectar las diferencias entre medias y el nivel de significancia $(\alpha)$ fue fijado en 0,05 . Los resultados son presentados como valores medios, error estándar y P-valor correspondiente.

\section{RESULTADOS}

La temperatura y humedad promedio para la sala de maternidad fueron de $28,7 \pm 4,5^{\circ} \mathrm{C}$ y $81 \pm 20 \%$ respectivamente, mientras que para la sala de gestación fueron de $27,6 \pm 3,42^{\circ} \mathrm{C}$ y $78,4 \pm 16 \%$.

La Tabla 3 muestra la influencia de la inclusión de distintos niveles de grasa sobre variables al nacimiento y al destete. Si bien no se encontraron diferencias estadísticamente significativas entre los tratamientos para el número de lechones nacidos vivos, muertos, totales y peso al nacimiento ( $p>0,05)$, una diferencia de \pm 2 lechones para estas variables, representa una diferencia considerable a nivel productivo.

El número y peso de lechones destetados se vio favorablemente influenciado por la inclusión de grasa durante el último tercio de gestación y la lactancia. Sin embargo, el IDC no se modificó por los tratamientos $(\mathrm{p}>0,05)$.

\section{DISCUSIÓN}

Se observó que la inclusión de grasa durante la gestación tardía (>90 días) y la lactación, influye favorablemente en el número y peso de lechones al destete. En contraste, dicha inclusión no afectó el IDC, LNV, LNT, LNM o el peso de la camada al nacimiento.

El aumento del número de lechones y su mayor peso al destete fue consistente con lo descrito en otra investigación ${ }^{11}$. Esto puede ser atribuido al incremento del contenido de grasa en la leche materna, el cual ha 
sido relacionado positivamente con la ganancia de peso de los lechones y el aumento de su supervivencia postnatal $1,7,21,24$.

En concordancia con estudios anteriores ${ }^{5,10,20}$, la adición de grasa no parece ejercer efecto sobre el número de LNV, LNT, LNM o el peso de la camada al nacimiento. Es importante señalar que si bien estadísticamente las diferencias no fueron significativas para el número de lechones nacidos vivos, en la Tabla 3 se aprecia una diferencia de hasta 2 lechones para los animales que consumieron grasa $(7 \%)$ con respecto al grupo control ( $0 \%$ ), lo cual significa -desde el punto de vista productivo- una mejoría en la eficiencia de las cerdas que recibieron mayor aporte de grasa en sus dietas hacia el final de la gestación

Respecto al IDC, no se encontraron diferencias entre los tratamientos. Parece ser que el factor más influyente para esta variable es la temperatura a la que están sometidas las cerdas. Otros autores señalaron que la adición de grasa durante la lactación no afectó el intervalo destete-celo en condiciones de termo-neutralidad, pero mostró una disminución de 8,3 días para cerdas mantenidas en ambientes de altas temperaturas ${ }^{4}$. Mas trabajos sobre esta temática deberían ser llevados a cabo, dado que la relación entre la adición de grasa y la performance subsecuente de las hembras, no están del todo claras ${ }^{22}$.

En conclusión, la adición de grasa durante la gestación tardía y la lactación no tuvo efecto sobre variables al nacimiento, como el número de lechones nacidos vivos, muertos, totales y peso de la camada. Tampoco afectó el intervalo destete-celo. Sin embargo, el peso y número de lechones al destete se vio favorablemente influenciado cuando se incorporó grasa a la dieta.

Agradecimientos. Al Ing ${ }^{\circ}$ Guillermo Arriortua y al personal de campo, por colaborar en el trabajo. Al INTA y la Asociación Cooperadora del INTA Las Breñas por el financiamiento de los insumos empleados, así como al CONICET por la beca doctoral que posibilitó esta investigación.

\section{REFERENCIAS}

1. Averette LA, Odle J, Monaco MH, Donovan SM. 1999. Dietary fat during pregnancy and lactation increases milk fat and insulin-like growth factor I concentrations and improves neonatal growth rates in swine. J Nutr 129: 21232129.

2. Black JL, Mullan BP, Lorschy ML, Giles LR. 1993. Lactation in the sow during heat stress. Liv Prod Sci 35: 153-170.

3. Boddicker RL et al. 2014. Gestational heat stress alters postnatal offspring body composition indices and metabolic parameters in pigs. PLoS One 9: 11 - el10859.

4. Cox NM, Britt JH, Armstrong WD, Alhusen HD. 1983. Effect of feeding fat and altering weaning schedule on rebreeding in primiparous sows. J Anim Sci 56: 21-29.
5. Holness DH, Mandisodza KT. 1985. The influence of additional fat in the diet of sows before and after parturition on piglet viability and performance. Livestock Prod Sci 13: 191-198.

6. Johnson JS. 2015. Thermal stress alters post-absorptive metabolism during pre-and postnatal development. En: Climate change impact on livestock: adaptation and mitigation, Springer, New Delhi, p. 61-79.

7. Lauridsen C, Danielsen V. 2004. Lactational dietary fat levels and sources influence milk composition and performance of sows and their progeny. Livestock Prod Sci 91: 95-105.

8. Lucy MC, Safranski TJ. 2017. Heat stress in pregnant sows: thermal responses and subsequent performance of sows and their offspring. Molec Reprod \& Dev 84: 946956.

9. National Research Council. 2012. Nutrient requirements of swine, The National Academies Press, $11^{\text {th }}$ revised ed., Washington DC.

10. Neal SM, Irvin KM, Shurson GC, Harris B, Hatfield EE. 1999. Effect of lactation diet fat level on sow and litter performance. Prof Anim Sci 15: 7-13.

11. Pettigrew JE. 1981. Supplemental dietary fat for peripartal sows: a review. J Anim Sci 53: 107-117.

12. Pettigrew JE, Moser RL. 1991. Fat in swine nutrition. En: Swine Nutrition, Ed. Miller, Ullrey \& Lewis, Stoneham, UK, p. 133-145.

13. Pierre NR, Cobanov B, Schnitkey G. 2003. Economic losses from heat stress by US livestock industries. J Dairy Sci 86: E52-E77.

14. Pollmann DS. 2010. Seasonal effect on sow herds: industry experience and management strategies. Midwest American Society of Animal Science Conference Proceedings, Des Moines, IA.

15. Quiniou N, Noblet J. 1999. Influence of high ambient temperatures on performance of multiparous lactating sows. J Anim Sci 77: 2124-2134.

16. Quiniou N, Richard S, Mourot J, Etienne M. 2008. Effect of dietary fat or starch supply during gestation and/ or lactation on the performance of sows, piglets' survival, and on the performance of progeny after weaning. Animal $2: 1633-1644$.

17. Renaudeau D et al. 2012. Adaptation to hot climate and strategies to alleviate heat stress in livestock production. Animal 6 : 707-728.

18. Renaudeau D et al. 2014. Selection for residual feed intake in growing pigs: effects on sow performance in a tropical climate. J Anim Sci 92: 3568-3579.

19. Rosero DS et al. 2012. Response of the modern lactating sow and progeny to source and level of supplemental dietary fat during high ambient temperatures. J Anim Sci 90 : 2609-2619.

20. Rosero DS et al. 2012. Sow and litter response to supplemental dietary fat in lactation diets during high ambient temperatures. J Anim Sci 90: 550-559.

21. Rosero DS et al. 2015. Impact of dietary lipids on sow milk composition and balance of essential fatty acids during lactation in prolific sows. J Anim Sci 93: 2935-2947. 
22. Rosero DS et al. 2016. Optimizing dietary lipid use to improve essential fatty acid status and reproductive performance of the modern lactating sow: a review. J Anim Sci \& Biotechnol 7: 34.

23. Santos OE et al. 2013. Effect of different systems for the control of environmental temperature on the performance of sows and their litters. Acta Sci Vet 41: 1.
24. Tilton SL et al. 1999. Addition of fat to the diets of lactating sows: I. Effects on milk production and composition and carcass composition of the litter at weaning. J Anim Sci 77: 2491-2500.

25. Williams AM et al. 2013. Effects of a controlled heat stress during late gestation, lactation, and after weaning on thermoregulation, metabolism, and reproduction of primiparous sows. J Anim Sci 91: 2700-2714. 